\title{
Advances in Magnetofection - Magnetically Guided Nucleic Acid Delivery: a Review
}

\author{
Sandeep Arora \\ Chitkara College of Pharmacy, Chitkara University, \\ Punjab, INDIA \\ Email: sandeep.arora@chitkara.edu.in \\ Girish Gupta \\ Omni Active Health Technologies, Thane, \\ Maharashtra, INDIA \\ Sukhbir Singh and Neelam Singh \\ Chitkara College of Pharmacy, Chitkara University, \\ Punjab, INDIA
}

\begin{abstract}
During the last decade, nanomagnetic methods for delivering and targeting nucleic acids have been developed which are often referred to as magnetofection. Nucleic acids carry the building plans of living systems. As such, they can be exploited to make cells produce a desired protein, or to shut down the expression of endogenous genes or even to repair defective genes. Hence, nucleic acids are unique substances for research as well as therapy to exploit their potential, they need to be delivered into cells which can be a challenging task in many respects. Magnetofection provides a novel tool for high throughput gene screening in vitro and can help to overcome fundamental limitations to gene therapy in vivo. Magnetofection is nucleic acid delivery to cells, supported and site-specifically guided by the attractive forces of magnetic fields acting on nucleic acid shuttles (vectors) which are associated with magnetic nanoparticles. In a magnetofection procedure, self-assembling complexes of enhancers like cationic lipids with plasmid DNA and small interfering RNA (siRNA) are associated with magnetic nanoparticles and are then concentrated at the surface of cultured cells by applying a permanent inhomogeneous magnetic field.
\end{abstract}

Keywords: Magnetofection, Magnetic Nanoparticles, Nucleic Acid Therapy,

Magnetic Drug Targeting.

Journal of Pharmaceutical Technology, Research and Management

Vol. 1

May 2013

pp. 19-29

agnetofection is defined as the magnetically enhanced delivery of
nucleicacids associated with magnetic nanoparticles. Magnetofection
is a novel, simple and highly efficient method to transfect cells
in culture. This method attempts to unite the advantages of the popular
biochemical (cationic lipids or polymers) and physical (electroporation,
gene gun) transfection methods in single system while excluding their
inconveniences including low efficiency and toxicity (Boussif et. al., 1995; agnetofection is defined as the magnetically enhanced delivery of
nucleicacids associated with magnetic nanoparticles. Magnetofection
is a novel, simple and highly efficient method to transfect cells
in culture. This method attempts to unite the advantages of the popular
biochemical (cationic lipids or polymers) and physical (electroporation,
gene gun) transfection methods in single system while excluding their
inconveniences including low efficiency and toxicity (Boussif et. al., 1995;

agnetofection is defined as the magnetically enhanced delivery of
nucleicacids associated with magnetic nanoparticles. Magnetofection
is a novel, simple and highly efficient method to transfect cells
in culture. This method attempts to unite the advantages of the popular
biochemical (cationic lipids or polymers) and physical (electroporation,
gene gun) transfection methods in single system while excluding their
inconveniences including low efficiency and toxicity (Boussif et. al., 1995;

agnetofection is defined as the magnetically enhanced delivery of
nucleicacids associated with magnetic nanoparticles. Magnetofection
is a novel, simple and highly efficient method to transfect cells
in culture. This method attempts to unite the advantages of the popular
biochemical (cationic lipids or polymers) and physical (electroporation,
gene gun) transfection methods in single system while excluding their
inconveniences including low efficiency and toxicity (Boussif et. al., 1995;

agnetofection is defined as the magnetically enhanced delivery of
nucleicacids associated with magnetic nanoparticles. Magnetofection
is a novel, simple and highly efficient method to transfect cells
in culture. This method attempts to unite the advantages of the popular
biochemical (cationic lipids or polymers) and physical (electroporation,
gene gun) transfection methods in single system while excluding their
inconveniences including low efficiency and toxicity (Boussif et. al., 1995; 
Arora, S Gupta, G. Singh, $S$ Singh N.
Cotton et. al., 1994). Magnetofection was invented by Christian Plank and Christian Bergemann and is registered as a trademark. It exploits magnetic force exerted upon gene vectors associated with magnetic particles to draw the vectors towards, possibly even into, the target cells. In this manner, the full vector dose applied gets concentrated on the cells within a few minutes so that $100 \%$ of the cells get in contact with a significant vector dose (Hughes et. al., 2001; Kanof et. al., 1999; Kasahara et. al., 1994).

\subsection{Steps of Magnetofection Process}

Magnetofection process used for enhanced delivery of biomolecules includes various steps such as:

- Biomolecules, such as nucleic acids including plasmid DNA and siRNA, are mixed with magnetic nanoparticles made of biodegradable iron oxides coated with cationic molecules in a simple one-step process to form a biomolecule / magnetic nanoparticle complex.

- The biomolecule / magnetic nanoparticle complex is added to the cells to be transfected in a standard multi-well plate.

- A magnetic force is applied beneath the cells to be transfected using a magnet array beneath the multi-well plate. This draws the biomolecule / magnetic nanoparticle complex onto cells on the bottom of the multi-well plate. The biomolecule is drawn towards, and delivered into, target cells, generally via endocytosis.

- This leads to rapid, efficient transfection without disturbing membrane architecture, causing chromosomal damage or leaving holes in cell membranes, therefore maintaining higher levels of cell viability. This is in stark contrast to physical transfection methods, including electroporation or biolistic methods, that can damage, create holes or electroshock the cell membranes causing cell death.

- The magnetic nanoparticles are biodegradable and non-toxic at the recommended doses.

- After delivery into the cells, the biomolecules are released into the cytoplasm by different mechanisms depending on the formulation used.

NanoTherics magnefect technology is unique in that the magnetic field created is designed to oscillate, which promotes more efficient uptake of the biomolecule into the cell and results in transfection efficiencies higher than other transfection techniques (Fouriki et. al., 2010; Hiemenz et. al., 2010; Finsinger et. al., 2000;) 


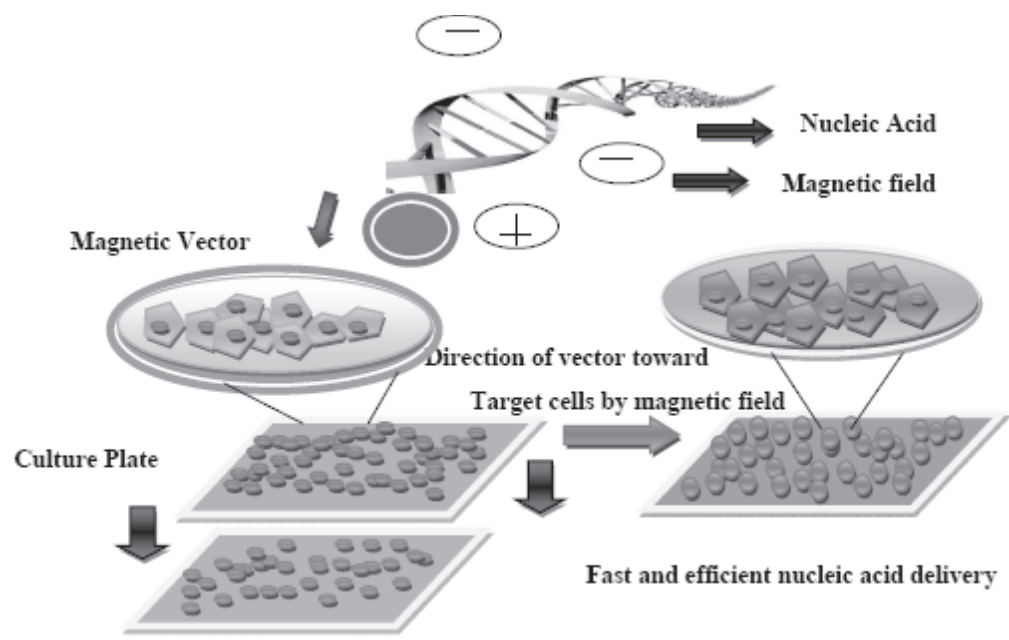

Advances in Magnetofection Magnetically Guided Nucleic Acid Delivery: a Review Magnetic Plate

Figure 1: Principle of Magnetofection in Cell Culture (Schillinger et. al., 2005)

\subsection{Principle of Magnetofection}

The magnetofection principle is to associate nucleic acids with cationic magnetic nanoparticles. These molecular complexes are then concentrated and transported into cells supported by an appropriate magnetic field (Isner et. al., 1996; Krüger et. al., 1994). Polyelectrolyte-coated magnetic nanoparticles are mixed with naked nucleic acids or synthetic or viral nucleic acid vectors in salt-containing buffer.

The particles associate with nucleic acids and vectors by electrostatic interaction and/or salt-induced colloid aggregation. The mixtures are added to cells in culture. The cell culture plate is positioned on a magnetic plate during 5-30 min of incubation. The magnetic field (s) rapidly sediment vectors on the cells to be transfected/transduced. In this way, the magnetic force allows a very rapid concentration of the entire applied vector dose onto cells, so that $100 \%$ of the cells get in contact with a significant vector dose.

\subsection{Mechanism of Magnetofection}

The magnetic nanoparticles are made of iron oxide, which is fully biodegradable, coated with specific cationic proprietary molecules varying upon the applications. Their association with the gene vectors (DNA, siRNA, virus, etc.) is achieved by salt-induced colloidal aggregation and electrostatic interaction. The magnetic particles are then concentrated on 
Arora, S Gupta, G. Singh, S Singh N. the target cells by the influence of an external magnetic field generated by magnets. The cellular uptake of the genetic material is accomplished by endocytosis and pinocytosis, two natural biological processes. Consequently, membrane architecture \& structure stays intact, in contrast to other physical transfection methods that damage the cell membrane (Ma et. al., 2011; Fouriki et. al., 2010; Hiemenz et. al., 2010; Finsinger et. al., 2000).

The nucleic acids are then released into the cytoplasm by different mechanisms depending upon the formulation used:

1) Proton sponge effect caused by cationic polymers coated on the nanoparticles that promote endosome osmotic swelling, disruption of the endosom membrane and intracellular release of DNA form.

2) Destabilization of endosome by cationic lipids coated on the particles that release the nucleic acid into cells by flip-flop of cell negative lipids and charge neutralization.

3) Usual viral infection mechanism when virus is used. Magnetofection works for primary cells and hard to transfect cells that are not dividing or slowly dividing, meaning that the genetic materials can go to the cell nucleus without cell division. Coupling magnetic nanoparticles to gene vectors of any kind results in a dramatic increase of the uptake of these vectors and consequently high transfection efficiency.

4) Mechanism of oscillating nanomagnetic transfection

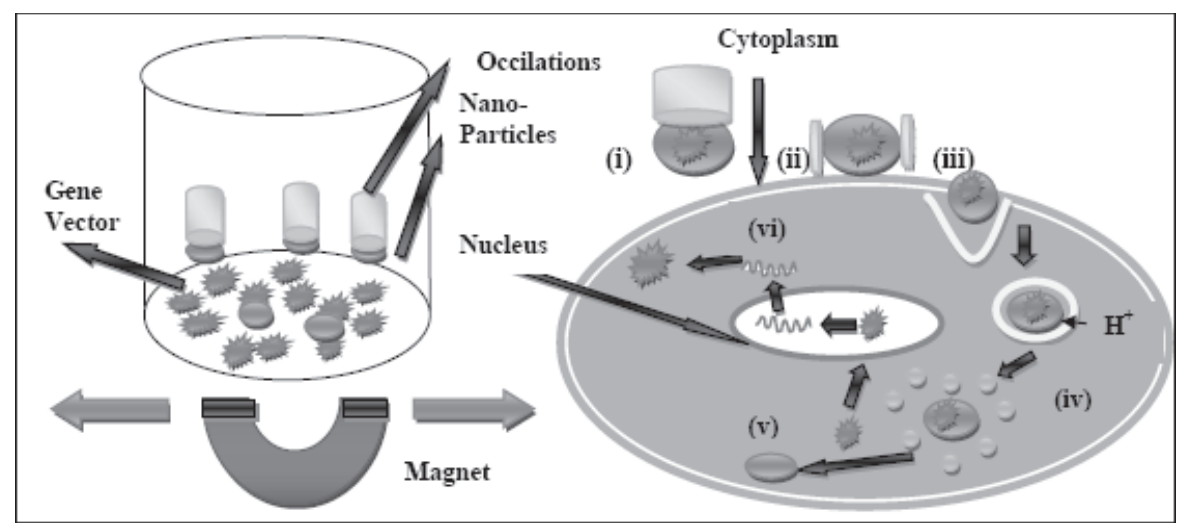

Figure 2: Proposed mechanism of oscillating nanomagnetic transfection (Fouriki et. al., 2010; Schillinger et. al., 2005) 
Plasmid DNA or siRNA is attached to magnet nanoparticles and incubated with cells in culture (left) (i)

An oscillating magnet array below the surface of the cell culture plate pulls the particle into contact with the cell membrane (ii) and drags the particles from side-to-side across the cells, (iii) mechanically stimulating endocytosis (iv) Once the particle/DNA complex is endocytosed, proton sponge effects rupture the endosome (v) releasing the DNA (vi) which then transcribes the target protein.

\subsection{Technology: Magnetofection Reagents}

(Li et. al., 1999; Leon et. al., 1998; Leventis et. al., 1990)

As the manufacturer of the magnetofection technology, chemicell offers two types of ready-to-use Magnetofection reagents.

PolyMAG is a universally applicable magnetic particle preparation for high efficiency nucleic acid delivery. It is mixed in a one-step procedure with the nucleic acid to be transfected and has been used successfully with plasmid DNA, antisense oligonucleotides and siRNAs.

CombiMAG is a magnetic particle preparation designed to be combined with any commercially available transfection reagent such as polycations and lipids and can be associated with plasmid DNA, antisense oligonucleotides, siRNAs or viruses. It allows you to create your own magnetic gene vector based on your favourite transfection reagent.

\subsection{Advantages of Magnetofection}

(Lu“bbe et. al., 1998; Lu bbe et. al., 1996; Mendenhall et. al., 1996)

Greatly improved transfection rates in terms of percentage of cells transfected compared to standard transfection.

- Up to several thousand fold increased levels of transgene expression compared to standard transfections upon short-term incubation.

- High transfection rates and transgene expression levels are achievable with extremely low vector doses, which allow saving expensive transfection reagents.

- Extremely short process time. A few minutes of incubation of cells with gene vectors are sufficient to generate high transfection efficiency, compared to several hours with standard procedures.

- Magnet assisted transfection, or Magnetofection ${ }^{\mathrm{TM}}$, is a simple, highly effective method to transfect cells.

Journal of Pharmaceutical Technology, Research and Management (JPTRM), Volume 1, May 2013
Advances in Magnetofection Magnetically Guided Nucleic Acid Delivery: a Review 
Arora, S Gupta, G. Singh, $S$ Singh N.
- It is an easy-to-use, fast and efficient technology that promotes the uptake of biomolecules, such as nucleic acids including plasmid DNA and siRNA, into cells using magnetic nanoparticles and magnetic fields.

- In cell culture, magnetic vectors are magnetically sedimented on the target cells within minutes. Thus, the diffusion barrier to nucleic acid delivery is overcome, the full vector dose comes in contact with the target cells, and introduction of genetic material is synchronized.

- Nucleic acid delivery is greatly accelerated and its efficiency with many, if not most, vector types is improved. Magnetofection is applicable to small and large nucleic acids.

- Low-dose requirements, the possibility of confining nucleic acid introduction to a localized area (magnetic targeting), and the amenability to high-throughput automation. Due to the favorable dose-response profile and the rapid kinetics, vector-related toxicity can be kept low.

- Magnetic nanoparticles for delivery of nucleic acid to target cells can use either non-viral or viral vectors.

\subsection{Applications of Magnetofection}

This has several important consequences:

- Magnetofection can be utilized to deliver synthetic siRNAs to cultured cells. Certain magnetic nanomaterials associate with siRNAs and are suitable for siRNA delivery, either alone or in combination with cationic polymers or cationic lipid enhancers; these complexes are targeted to the

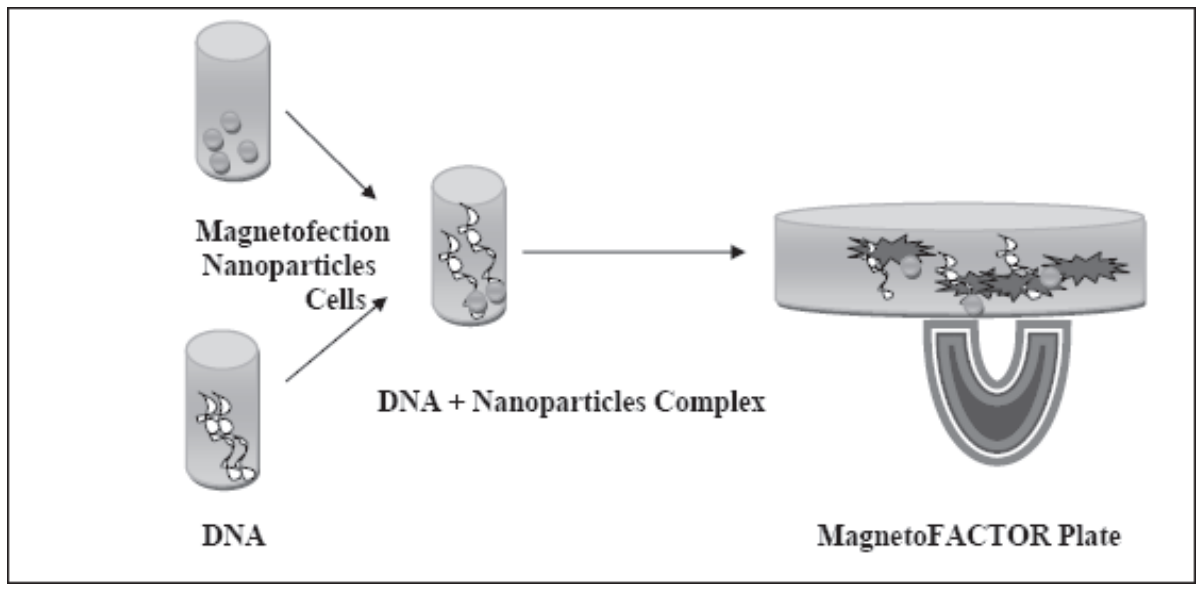

Figure 3: Magnetic nanomaterials associated with DNA (http://www.bocascientific.com)

Journal of Pharmaceutical Technology, Research and Management (JPTRM), Volume 1, May 2013 


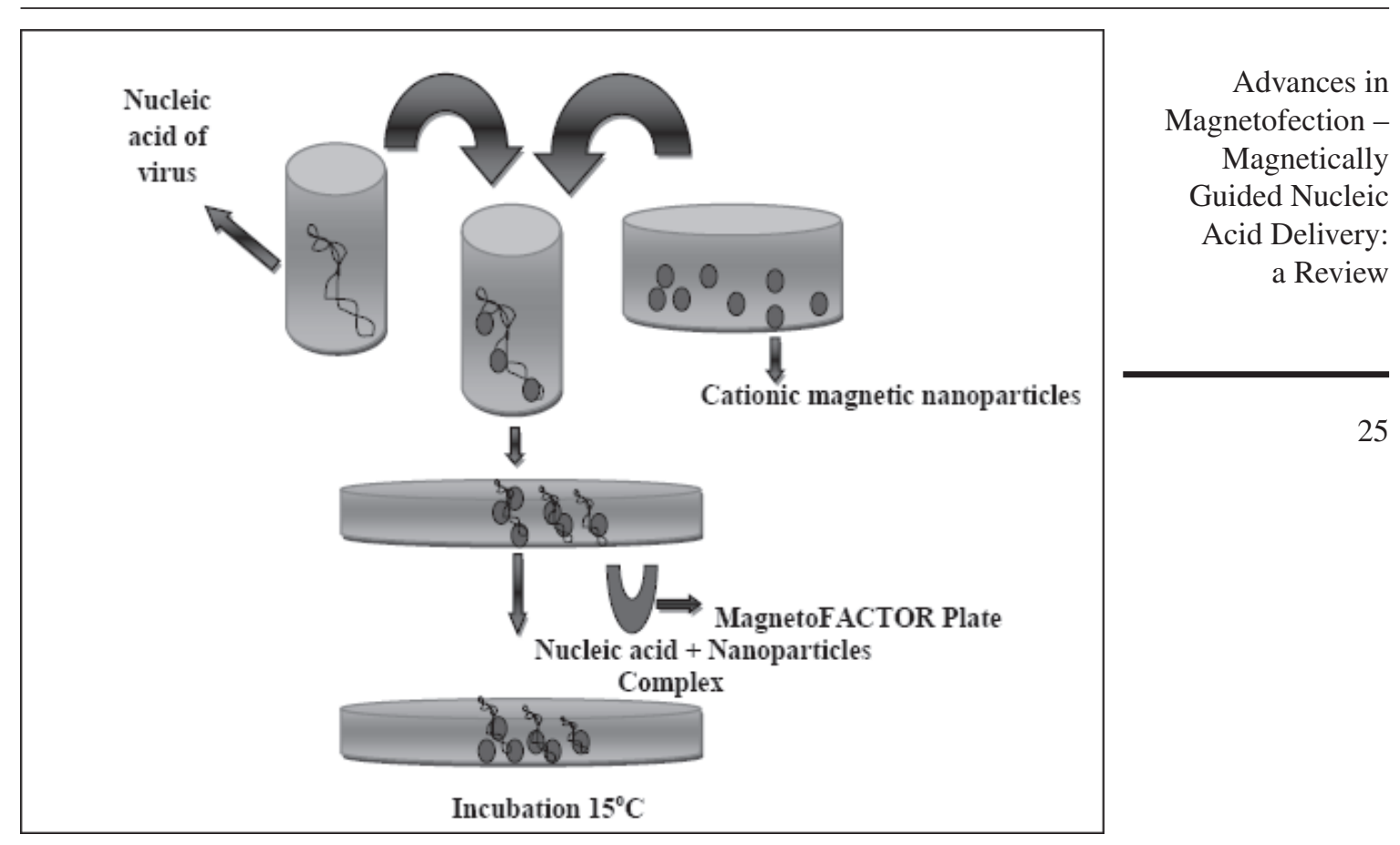

Figure 4: Magnetofection for Virtal DNA transfection (www.chemicell.com; www. ozbiosciences.com)

cell surface by application of a gradient magnetic field (Campbell et. al., 2012; Mair et. al., 2009; Luo et. al., 2000)

- Magnetofection is used for nucleic acid delivery by magnetic force acting on magnetic particles and nucleic acids or nucleic acid vectors. Vectors are bound to magnetic, usually iron oxide, nanoparticles, in most cases by non-covalent bonds. Magnetic force accumulates and/or holds magnetic vectors in a target tissue against hydrodynamic forces (Mair et. al., 2009; Luo et. al., 2000; Widder et. al., 1983).

- Magnetofection has been adapted to all types of nucleic acids (DNA, siRNA, dsRNA, shRNA, mRNA, ODN), non-viral transfection systems (transfection reagents) and viruses. It has been successfully tested on a broad range of cell lines, hard-to-transfect and primary cells (Mykhaylyk et. al., 2008; Tomko et. al., 1997; Plank et. al., 1994).

- Ocean NanoTech has developed superparamagnetic nanocrystals made of iron oxide that are $5 \mathrm{~nm}$ to $50 \mathrm{~nm}$ in diameter that are ideal for magnetofection. The surfaces of the superparamagnetic nanocrystals are modified with biocompatible polymers with specific proprietary 
Arora, S Gupta, G. Singh, $S$ Singh N. anionic and cationic molecules that vary upon the applications. The superparamagnetic nanocrystals carrying the gene vectors are used to transfect the target cells through the influence of an external magnetic field. The cellular uptake of the genetic material is accomplished by two natural biological processes, endocytosis and pinocytosis, induced by the applied magnetic field. Use of the 5 or $10 \mathrm{~nm}$ superparamagnetic nanocrystals from Ocean NanoTech allows the membrane architecture \& structure to remain intact unlike other bigger nanoparticles and physical transfection methods that damage the cell membrane. Small DNA sequences and plasmid are being introduced to the iron oxide nanocrystals which will subsequently be used to transfect specific cells (Pickles et. al., 2000; Meyer et. al., 1995; Wu et. al., 1987).

- New magnetic technologies have been developed to improve the uptake and expression of DNA and siRNA in cells growing in culture (Sukoyan et. al., 2013; Plank et. al., 2003; Plank et. al., 1999).

- Gene vectors were associated with super-paramagnetic nanoparticles and targeted gene delivery by application of a magnetic field. This potentiated the efficacy of any vector up to several hundred-fold, allowed reduction of the duration of gene delivery to minutes, extended the host tropism of adenoviral vectors to non-permissive cells and compensated for low retroviral titer (Scherer et. al., 2002; Povey et. al., 1986).

\subsection{Biodistribution of magnetic nanoparticles}

- The biodegradable cationic magnetic nanoparticles are not-toxic at the recommended doses and even at higher doses. Gene vectors / magnetic nanoparticles complexes are seen into cells after 10-15 minutes that is much faster than any other transfection method. After 24,48 or 72 hours, most of the particles are localized in the cytoplasm, in vacuoles (membranes surrounded structure into cells) and occasionally in the nucleus (Plank et. al., 2011; Scherer et. al., 2002; Shayakhmetov et. al., 2000; Povey et. al., 1986).

\section{CONCLUSION: FUTURE PERSPECTIVES OF MAGNETOFECTION}

A very exciting future perspectives of magnetofection is to use it therapeutically e.g. in tumor targeting or local neo-vessel formation. Apart from direct injection into the target tissue, the injection into blood vessels which are rather distant to the target site is assumed to become the most important form of vector administration for therapeutic magnetofection.

Journal of Pharmaceutical Technology, Research and Management (JPTRM), Volume 1, May 2013 
Iron oxide nanoparticles can be used as "superparamagnetic", which means that they are strongly attracted to a magnetic field but they do not retain residual magnetism after the field is removed. Therefore they cannot agglomerate (like ferromagnetic particles) after removal of the magnetic field. It has been found that iron oxide particles used as contrast agents in magnetic resonance imaging (MRI) are fully biocompatible.
Advances in Magnetofection Magnetically Guided Nucleic Acid Delivery: a Review

\section{REFERENCES}

Boussif, O. (1995) 'A versatile vector for gene and oligonucleotide transfer into cells in culture and in vivo - Polyethylenimine'. Proceedings of the National Academy of Sciences of the United State of America, Vol 92, pp. 7297-7301 http://dx.doi.org/10.1073/pnas.92.16.7297

Campbell, K. Calvo, C. J. Mironov, S. Herron, T. Berenfeld, O and Jalife, J. (2012) 'Spatial gradients in action potential duration created by regional magnetofection of hERG are a substrate for wavebreak and turbulent propagation in cardiomyocyte monolayers'. J Physiol., Vol 590, 24, pp. 6363-6379 http://dx.doi.org/10.1113/jphysiol.2012.238758

Cotton, M. (1994) 'Psoralen treatment of adenovirus particles eliminates virus replication and transcription while maintaining the endosomolytic activity of the virus capsid'. Virology, Vol 205, pp. 254-261 http://dx.doi.org/10.1006/viro.1994.1641

Curiel, D.T. (1999) 'Strategies to adapt adenoviral vectors for targeted delivery'. Annals of New York Academy of Sciences, Vol 886, pp.158-171 http://dx.doi.org/10.1111/j.1749-6632.1999.tb09409.x

Fasbender, A. (1998) 'Incorporation of adenovirus in calcium phosphate precipitates enhances gene transfer to airway epithelia in vitro and in vivo'. J Clin Invest, Vol 102, pp. 184-193 http://dx.doi.org/10.1172/JCI2732

Finsinger, D. (2000) 'Protective copolymers for nonviral gene vectors: synthesis, vector characterization and application in gene delivery'. Gene Therapy, Vol 7, pp.1183-1192 http://dx.doi.org/10.1038/sj.gt.3301227

Fouriki, A. Farrow, N. Clements, M.A. and Dobson, J. (2010) 'Evaluation of the magnetic field requirements for nanomagnetic gene transfection'. Nano Reviews, Vol 1, 10, pp. 1-5

Hiemenz, P.C. (1986) 'Principles of Colloid and Surface Chemistry' (2 $2^{\text {nd }}$ ed.) Marcel Dekker: New York, pp. 508-528

Hughes, C. Galea-Lauri, J. Farzaneh, F. and Darling, D. (2001) 'Streptavidin paramagnetic particles provide a choice of three affinity-based capture and magnetic concentration strategies for retroviral vectors'. Mol Therapy, Vol 3, pp. 623-630 http://dx.doi.org/10.1006/mthe.2001.0268

Isner, J.M. (1996) 'Arterial gene transfer for therapeutic angiogenesis in patients with peripheral artery disease'. Human Gene Therapy, Vol 20, pp. 959-988 http://dx.doi.org/10.1089/hum.1996.7.8-959

Kanof, M.E. Smith, P.D. and Zola, H. (1999) 'Preparation of human mononuclear cell populations and subpopulations, In: Coligan JE'. Current Protocols in Immunology. John Wiley: New York, pp. 7

Kasahara, N. Dozy, A.M. and Kan, Y.W. (1994) 'Tissue-specific targeting of retroviral vectors through ligand-receptor interactions'. Science, Vol 266, 5189, pp. 1373-1376 http://dx.doi.org/10.1126/science.7973726 
Arora, S

Gupta, G.

Singh, $S$

Singh N.
Kru" ger, A. Schirrmacher, V. and Von, H.P. (1994) 'Scattered micrometastases visualized at the single-cell level: detection and re-isolation of lacZ-labeled metastasized lymphoma cells'. International J Cancer, Vol 58, pp. 275-284 http://dx.doi.org/10.1002/ijc.2910580222

Leon, R.P. (1998) 'Adenoviral-mediated gene transfer in lymphocytes'. Proceedings of the National Academy of Sciences of the United State of America, Vol 95, pp. 13159-13164 http://dx.doi.org/10.1073/pnas.95.22.13159

Leventis, R. and Silvius, J.R. (1990) 'Interactions of mammalian cells with lipid dispersions containing novel metabolizable cationic amphiphiles'. Biochimica et biophysica acta, Vol 1023, pp. 124-132 http://dx.doi.org/10.1016/0005-2736(90)90017-I

Li, D. Duan, L. Freimuth, P. O’Malley and Jr, B.W. (1999) 'Variability of adenovirus receptor density influences gene transfer efficiency and therapeutic response in head and neck cancer'. Clinical Cancer Research, Vol 5, pp. 4175-4181

Lu"bbe, A.S. (1996) 'Clinical experiences with magnetic drug targeting: a phase I study with 4_epidoxorubicin in 14 patients with advanced solid tumors.' Cancer Research; Vol. 56, pp. 4686-4693

Lu"bbe, A.S. and Bergemann, C. (1998) 'Magnetically controlled drug targeting' The Cancer J, Vol 1, pp. 104-105

Luo, D. Saltzman, W.M. (2000) 'Enhancement of transfection by physical concentration of DNA at the cell surface'. Nature Biotechnology, Vol 18, pp. 893-895 http://dx.doi.org/10.1038/78523

Ma, Y. Zhang, Z. Wang, X. Xia, W and Gu, H. (2011) 'Insights into the mechanism of magnetofection using MNPs-PEI/pDNA/free PEI magnetofectins'. Intnl J Pharmaceutics. Vol 31, 419, pp. 247-54 http://dx.doi.org/10.1016/j.ijpharm.2011.07.017

Mair, L. (2009) 'Size-Uniform $200 \mathrm{~nm}$ Particles: Fabrication and Application to Magnetofection'. J Biomed Nanotechnology, Vol 5, 10, pp. 182-191 http://dx.doi.org/10.1166/jbn.2009.1024

Mendenhall, G.D. Geng, Y. and Hwang, J. (1996) 'Optimization of long-term stability of magnetic fluids from magnetite and synthetic polyelectrolytes'. J Colloid Interface Sciences, Vol 184, pp. 519-526 http://dx.doi.org/10.1006/jcis.1996.0647

Meyer, K.B. (1995) 'Intratracheal gene delivery to the mouse airway: characterization of plasmid DNA expression and pharmacokinetics'. Gene Therapy, Vol 2, pp. 450-460

Mykhaylyk, O. Zelphati, O. Rosenecker, J. and Plank, C. (2008) 'siRNA delivery by magnetofection' Current Opinion of Molecular Therapy, Vol 10, 5, pp. 493-505

Pickles, R.J. (2000) 'Retargeting the coxsackievirus and adenovirus receptor to the apical surface of polarized epithelial cells reveals the glycocalyx as a barrier to adenovirus-mediated gene transfer'. J Virology, Vol 74, pp. 6050-6057 http://dx.doi.org/10.1128/JVI.74.13.6050-6057.2000

Plank, C. (1994) 'The influence of endosome-disruptive peptides on gene transfer using synthetic virus-like gene transfer systems'. J Biological Chem, Vol 269, pp. 12918-12924

Plank, C. Anton, M. Rudolph, C. Rosenecker, J. and Krötz, F. (2003) 'Enhancing and targeting nucleic acid delivery by magnetic force'. Expert opinion on biological therapy, Vol 3, 5, pp.745-758 http://dx.doi.org/10.1517/14712598.3.5.745

Plank, C. Schillinger, U. and Scherer, F. (2003) 'The magnetofection method: using magnetic force to enhance gene delivery'. J Biol Chem, Vol 384, 5, pp. 737-747

Plank, C. Tang, M. Wolfe, A. and Szoka, F.C. (1999) 'Branched cationic peptides for gene delivery. Role of type and number of cationic residues in formation and in vitro activity of DNA polyplexes'. Human Gene Therapy, Vol 10, pp. 319-333 http://dx.doi.org/10.1089/10430349950019101

Journal of Pharmaceutical Technology, Research and Management (JPTRM), Volume 1, May 2013 
Plank, C. Zelphati, O and Mykhaylyk, O. (2011) 'Magnetically enhanced nucleic acid delivery. Ten years of magnetofection-progress and prospects'. Advanced Drug Delivery Reviews. Vol 63, 14, pp. 1300-1331 http://dx.doi.org/10.1016/j.addr.2011.08.002

Povey, A.C. Bartsch, H. Nixon, J.R. and O'Neill, I.K. (1986) 'Trapping of chemical carcinogens with magnetic polyethyleneimine microcapsules: I. Microcapsule preparation and in vitro reactivity of encapsulated nucleophiles'. J Pharm Sci, Vol 75, pp. 831-837 http://dx.doi.org/10.1002/jps.2600750902

Scherer, F. Anton, M. and Schillinger, U. (2002). 'Magnetofection: enhancing and targeting gene delivery by magnetic force in vitro and in vivo'. Gene Therapy, Vol 9, 2, pp. 102-109 http://dx.doi.org/10.1038/sj.gt.3301624

Schillinger, U. Brill, T. Rudolph, C. Huth, S. Gersting, S. and Krötz, F. (2005) 'Advances in magnetofection - magnetically guided nucleic acid delivery' $J$ Magnetism and Magnetic Material. Vol 293, pp. 501-508 http://dx.doi.org/10.1016/j.jmmm.2005.01.032

Shayakhmetov, D.M. Papayannopoulou, T. Stamatoyannopoulos, G. and Lieber, A. (2000) 'Efficient gene transfer into human CD34 (+) cells by a retargeted adenovirus vector'. J Virology, Vol 74, pp. 2567-2583 http://dx.doi.org/10.1128/JVI.74.6.2567-2583.2000

Sukoyan, M. A. Khrapov, E. A. Voronina, E. N. Boyarskikh, U. A. Gubanov, A. I. Itin, V. I. Magaeva, A. A. Nayden, E. P. Terekhova, O. G and Filipenko, M. L. (2013) 'Magnetofection of Human Somatic Cells with Magnetite and Cobalt Ferrospinel Nanoparticles'. Bulletin of Exp Biol and Med. Vol 154, 5, pp. 673-676 http://dx.doi.org/10.1007/s10517-013-2027-2

Tomko, R.P. Xu, R. and Philipson, L. (1997) 'HCAR and MCAR: the human and mouse cellular receptors for subgroup $\mathrm{C}$ adenoviruses and group B coxsackieviruses'. Proceedings of the National Academy of Sciences of the United State of America, Vol 94, pp. 3352-3354 http://dx.doi.org/10.1073/pnas.94.7.3352

Widder, K.J. (1983) 'Selective targeting of magnetic albumin microspheres to the Yoshida sarcoma: ultrastructural evaluation of microsphere disposition'. European J Cancer Clinical Oncology, Vol 19, pp.141-147 http://dx.doi.org/10.1016/0277-5379(83)90409-1

Wu, G.Y. and Wu, C.H. (1987) 'Receptor-mediated in vitro gene transformation by a soluble DNA carrier system'. The J Biol Chem, Vol 262, pp.4429-4432

<URL: http://www.bocascientific.com/transfectionmagnetofection-c-141.html>

<URL: http://www.chemicell.com/products/Magnetofection/Magnetofection_separation.html>

<URL: http://www.ozbiosciences.com/magnetofection.html>

\section{Magnetofection -}

Magnetically

Guided Nucleic

Acid Delivery:

a Review 